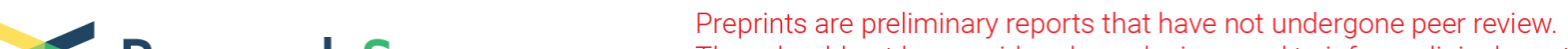 Research Square
They should not be considered conclusive, used to inform clinical practice,
or referenced by the media as validated information.
}

\section{A Retrospective Study of Prevalence of Porcine Parvoviruses 6 in Guangxi, China and Its Co- Infection with Porcine Circovirus}

\section{Ning Yu}

Changchun Veterinary Research Institute, Chinese Academy of Agricultural Sciences

\section{Ziping Yu}

Changchun Veterinary Research Institute, Chinese Academy of Agricultural Sciences

\section{Luer Chen}

Changchun Veterinary Research Institute, Chinese Academy of Agricultural Sciences

Yumeng Liu

Changchun Veterinary Research Institute, Chinese Academy of Agricultural Sciences

\section{Sheng Feng}

Changchun Veterinary Research Institute, Chinese Academy of Agricultural Sciences

\section{Xinyu Zhuang}

Changchun Veterinary Research Institute, Chinese Academy of Agricultural Sciences

\section{Zhuoxin Li}

Changchun Veterinary Research Institute, Chinese Academy of Agricultural Sciences

\section{Chenghui Li}

Changchun Veterinary Research Institute, Chinese Academy of Agricultural Sciences

\section{Xiangyu Zhu}

Changchun Veterinary Research Institute, Chinese Academy of Agricultural Sciences

\section{He Zhang}

Changchun Veterinary Research Institute, Chinese Academy of Agricultural Sciences

\section{Wei Wang ( $\square$ wwky1101@126.com)}

Changchun Veterinary Research Institute, Chinese Academy of Agricultural Sciences

\section{Wenchao Sun}

Changchun Veterinary Research Institute, Chinese Academy of Agricultural Sciences

\section{Ningyi Jin}

Changchun Veterinary Research Institute, Chinese Academy of Agricultural Sciences

\section{Huijin Lu}

Changchun Veterinary Research Institute, Chinese Academy of Agricultural Sciences

\section{Research Article}


Keywords: Porcine parvovirus, Porcine circovirus, phylogenetic analysis

Posted Date: March 3rd, 2022

DOI: https://doi.org/10.21203/rs.3.rs-1373424/v1

License: (c) (i) This work is licensed under a Creative Commons Attribution 4.0 International License. Read Full License 


\section{Abstract \\ Background}

Parvovirus can infect human and a variety of animals (such as pigs, dogs, cats, etc.). Porcine parvovirus is characterized by infertility, mummified fetuses, early embryonic death, and stillbirths. Worldwide, many porcine parvoviruses (PPVs) have been linked to porcine circovirus (PCV) associated disease (PCVAD).

\section{Results}

By PCR amplification and sequencing, the total PPV6-positive rate was 12.5\% (62/497) and the positive rate of PPV6 ranged from 12.0-17.6\% in differently city. Meanwhile, we found that the positive rate of PPV6 co-infection with PCV (69.4\%) was significantly higher than that of PPV6 infection alone (30.6\%) (p $<0.01)$. The positive rate of PPV6 co-infected with PCV2 was significantly higher than that of PPV6 coinfection with PCV3 and PCV4. Of ten PPV6 isolates, six isolates were $6112 \mathrm{nt}$ in length and four isolates were 6111 nucleotides (nt) in length. Compared with the reference strains, we found that the 3 ' UTRs of ten strains are lacked 14 and 15 bases, respectively, which leads to significant changes in the RNA secondary structure. Within the VP1 unique region, compared with PPV1, PPV2 and PPV3, the motifs of the $\mathrm{Ca}^{2+}$ binding loop (YTGPR) and the catalytic residues (HDIRY) of putative secretory PLA2 were very conserved without amino acid mutation. Phylogenetic analysis of the NS1 gene showed that all PPV6 isolates were located in the genus Copiparvovirus together with PPV4 and PPV5. Phylogenetic analysis of the VP1 gene showed that all PPV6 strains isolated from China clustered in group B, except for the three Chinese strains that clustered in group $A$. Combined with the results of multiple sequence alignment and genetic evolution, we found a total of 14 amino acid mutation related to phylogenetic tree branches (R14K/Q, I116L/K/T, N788S, R789/M, S/T791H, M792H, A815S, D873G, N919S, R938K, S941T, R1013H, T1038N, T1042D/Y). Compared with PPV6 sequences in different geographic regions, the VP1 gene is more prone to mutation than NS1 gene. From which, two adaptive mutations were found in NS1 (Q3H, P20H) and VP1 (A619T, Q730E), respectively. In addition, we predicted the B cell and T lymphoid epitopes of the NS1 and VP1 genes of 10 PPV6 strains, respectively, and speculated that compare with VP1, the NS1 gene is more suitable as an epitope vaccine target.

\section{Conclusion}

In summary, our findings provide new insight into the prevalence of PPV6 in Guangxi, China, as well as lay the foundation for the study of virus infection mechanism.

\section{Background}

The family Parvoviridae consists of two subfamilies: the Parvovirinae, which infect vertebrates, and the Densovirinae, which infect invertebrates [1]. Parvovirinae is currently proposed to be classified into eight 
genera by the International Committee on the Taxonomy of Virus (ICTV): Protoparvovirus, Bocaparvovirus, Copiparvovirus, Tetraparvovirus, Erythropavovirus, Amdoparvovirus, Aveparvovirus, and Dependoparvovirus [2]. First four genera above contain viruses that infect pigs [3]. Parvoviruses are small, single-stranded, non-enveloped DNA virus approximately 4-6.3 kb in length, containing two major open reading frames (ORFs). Translation of the ORF1 encodes non-structural protein (NSP) involved in viral replication; Translation of the ORF2 encodes capsid protein (VP1) [4, 5].

Parvovirus is a ubiquitous virus in nature, which has a wide range of hosts, for example, parvovirus B19 (humans: infectious erythema), canine parvovirus (dogs: viral or hemorrhagic enteritis), feline parvovirus (cats: Panleukopenia), porcine parvovirus (pigs: mainly reproductive disorders) [3, 6]. At the end the 1960s, Porcine parvovirus (PPV1) was first recognized as a member of the Parvoviridae family, and have confirmed that it was the causative agent of SMEDI syndrome (Stillbirths, mummification, embryonic death and infertility, SMEDI) [7-9]. During the past decade, with the use of molecular assays and pathogen discovery tools, a number of novel parvoviruses belonging to the subfamily parvovirinae have been identified in pigs, including tetraparvovirus (PPV2-3) and copiparvovirus (PPV4-6) [10]. In addition, in 2016, Palinski first discovered PPV7, in the rectal swabs of healthy adult pigs by macrogenomic sequencing. The NS1 protein of PPV7 have less than $30 \%$ identity to other genera, leading to it inclusion in the genus Chapparvovirus [11]. Since its discovery, PPV has remained a constant worldwide problem of the pig industry, and remains one of the most common and important infectious agents of infertility.

PPV1 has been well known to pig producers for decades and is mainly associated with reproductive failure manifest as infertility, mummified fetuses, early embryonic death, and stillbirths [6]. Porcine circovirus type 2 (PCV2) is small single stranded circular DNA virus which is related to many disease manifestations in pigs of all ages collectively summarized as PCV associated disease (PCVAD) [12]. Depending on the different pathogen it is co-infected with, the main symptoms are PMWS, respiratory disease, diarrhea, stillborn, mummified, porcine dermatitis and nephropathy syndrome (PDNS) [13]. In 2014, researchers confirmed that co-infection of porcine parvovirus and porcine circovirus type2 is prevalent, indicating their cooperative roles in porcine circovirus-associated diseases [14]. Recent research has indicated concurrent infection with PCV2 and PPV increases disease and lesion severity compared to mono-infection with PCV2 [15]. PPV6 was first identified in aborted pig fetuses in China in 2014 and subsequently was also reported in the USA [1]. Later, it was reported that PPV6 could coinfection with PCV2 and PRRSV $[12,16,17]$. Retrospective research findings that at least 2 countries have found PPV6 in their porcine populations, including America and Poland [10,17]. Although inactivated vaccine and live attenuated vaccine are widely used in pig industry, the outbreak of PPV epidemic in the world is still increasing year by year, seriously endangering the development of pig industry. Timely detection new pathogens are of great significance to control and prevention of diseases.

The purpose of this study was to assess the prevalence of PPV6 and its co-infection with parvovirus in Guangxi, China. At the same time, a comprehensive genetic evolution analysis was performed based on NS1 and VP1 of porcine parvovirus, which laid the foundation for subsequent research. 


\section{Methods}

\section{Sample information}

From 2015 to 2020, Serum samples $(n=497)$ were collected from 2 to 17-week-old pigs located on nine pig farms designated as Nanning, Baise, Yulin, Fang Chenggang (FCG), Beihai, Qinzhou, Hechi, and Laibin (Fig. 1). Serum samples used in this study were obtained from the Guangxi Center for Animal Disease Prevention and Control. The serum samples are stored at $-40^{\circ} \mathrm{C}$ until testing. The experiment was approved by the Animal Welfare and the Animal Experimental Ethical Committee (Guangxi University, No. Xidakezi2000138) (see Ethics approval and consent to participate).

\section{DNA extraction and polymerase chain reaction (PCR)}

The serum samples were separated from the collected blood, followed by viral DNA extraction. Total DNA was extracted from $100 \mu$ l of serum using the TIANamp Virus DNA Kit (TIANGEN, Beijing, China) following the manufacturer's directions. Six primer pairs were designed based on the reference sequence from NCBI (Supplementary Table S1). The PCRs were carried out in 96-well plates, with each mixture containing a total volume of $25 \mu \mathrm{L}, 1 \mu \mathrm{L}$ of extracted DNA, $1 \mu \mathrm{L}$ of primer pairs $(10 \mu \mathrm{M}), 12.5 \mu \mathrm{L}$ of $2 \times$ pfu MasterMix (Dye) (Vazyme, Nanjing, China), and $10.5 \mu \mathrm{L}$ of RNase-free water. The PCR amplification reactions were performed using Nucleic Acid Electrophoresis under universal conditions: predenaturation for 3 minutes at $94^{\circ} \mathrm{C}$, followed by 30 cycles of 30 seconds at $94^{\circ} \mathrm{C}, 30$ seconds at $56^{\circ} \mathrm{C}$, an extension for 2 minutes at $72^{\circ} \mathrm{C}$, and a final extension for 10 minutes at $72^{\circ} \mathrm{C}$. Subsequently, the amplified PCR products were confirmed by agarose gel electrophoresis. The specific DNA bands purified by the Biospin Gel Extraction Kit (Hangzhou Bire Co. Hangzhou, China). PMD19-T vector (Takara Co. Dalian, China) were used to construct recombinant vectors, which were amplified in Escherichia coli Trans-T1 competent cells. The positive amplicons were sequenced at Comate Bioscience Co. Ltd. Forward and reverse sequencing were done.

\section{Co-infection detection}

The DNA from PPV6-positive serum samples was selected for the detection of PCV2, PCV3 and PCV4, using the published primers and protocols as those previously described. (Supplementary Table S2).

\section{Phylogenetic analysis}

The genome sequences of PPV6 obtained in this study have been deposited in GenBank under the accession numbers OM811448-OM811457. The sequences were assembled using SeqMan software (DNASTAR Inc., Madison, Wisconsin, USA) and aligned using Molecular Evolutionary Genetics Analysis (MEGA 7.0) software with the Clustal W alignment method for genomic similarity analysis. A phylogenetic analysis of the complete PPV6 genome was reconstructed using MEGA 7.0 software with maximum-likelihood (ML) method and neighbor joining $(\mathrm{NJ})$ method with 1000 bootstrap replicates. In addition to the PPV 6 viruses, the complete sequences of various other parvoviruses were obtained from GenBank. (Supplementary Table S3). 


\section{Other online software}

RNA secondary structure of PPV6 isolates were predicted using online program RNA fold web server (http://rna.tbi.univie.ac.at/cgi-bin/RNAWebSuite/RNAfold.cgi). T lymphocyte CTL epitopes of PPV6 isolates were predicted using the ComPred online program of nHLAPred ComPred (http://crdd.osdd.net/raghava/nhlapred/). The threshold was adjusted to 0.5 to predict binding peptides for five different molecules, HLA-A2, HLA-A*0201, HLA-A*0202, HLA-A*0203 and HLA-A*0205, respectively. $B$ lymphocyte epitopes of PPV6 isolates were predicted using the online program IEDB (http://www.immuneepitope.org/tools/bcell/iedb_input). Higher score of the peptide means the higher probability to be as epitope.

\section{Results}

\section{Detection of PPV6 and PCV2, PCV3 and PCV4}

In the present study, among the 497 serum samples from nine different pig farms. The positive rate of PPV6 in these samples were 12.5\% (62/497). It is noteworthy that the positive rate of PPV6 ranged from $12-17.6 \%$, with the highest rate recorded in Hechi, and the lowest in Beihai. Additionally, the positive rate of PCV in these samples were $8.7 \%$ (43/497) (Table 1$)$.

Table 1

Detection of PPV6 co-infections with PCV2, PCV3 and PCV4 in serum samples isolates from nine cities in Guangxi, China.

\begin{tabular}{|llllll|}
\hline Prefecture & Number of & PPV6 & \multicolumn{2}{l}{ Co-infection } \\
\cline { 6 - 7 } & samples & positive & PCV2 & PCV3 & PCV4 \\
\hline Nanning & 103 & $13 / 103$ & $7 / 13$ & $3 / 13$ & $1 / 13$ \\
\hline Baise & 58 & $6 / 58$ & $2 / 6$ & $1 / 6$ & $0 / 6$ \\
\hline Yulin & 75 & $9 / 75$ & $6 / 9$ & $3 / 9$ & $0 / 9$ \\
\hline Guigang & 39 & $7 / 39$ & $3 / 7$ & $0 / 7$ & $0 / 7$ \\
\hline Fang Chenggang & 62 & $5 / 62$ & $0 / 5$ & $1 / 5$ & $0 / 5$ \\
\hline Beihai & 25 & $3 / 25$ & $1 / 3$ & $1 / 3$ & $0 / 3$ \\
\hline Qinzhou & 41 & $4 / 41$ & $2 / 4$ & $0 / 4$ & $1 / 4$ \\
\hline Hechi & 85 & $15 / 85$ & $8 / 15$ & $3 / 15$ & $0 / 15$ \\
\hline Laibin & 9 & $0 / 9$ & 0 & 0 & 0 \\
\hline Total & 497 & $62 / 497$ & $29 / 62$ & $12 / 62$ & $2 / 62$ \\
\hline
\end{tabular}


Among the 62 PPV6-positive samples, co-infection of PPV6 with porcine circovirus, including PCV2, PCV3 and PCV4 was analyzed. In this study, the co-infection rate of PPV6 and PCV was $69.4 \%$ (43/62). It is indicated that the positive rate of PPV6 co-infection with PCV was significantly higher than that of PPV6 infection alone (30.6\%) ( $\mathrm{P}<0.01)$. The PPV6 co-infections with PCV2, PCV3 and PCV4 were detected in $46.8 \%$ (29/62), $19.4 \%$ (12/62), 3.2\% (2/62) of the samples, respectively. These data indicate that a high co-infection rate of PPV6 with PCV2 and PCV3 exist, which provide valuable information for further study into the pathology of PPV6 in association with PCV2 and PCV3.

\section{Sequence analysis of the complete genome sequences of ten PPV6 isolates}

To analyze the genetic relationship of PPV6 strains collected in Guangxi, 10 complete sequences of the PPV6 genome were amplified and sequenced. From which, six isolates (PPV6/GX05, PPV6/GX41, PPV6/GX33, PPV6/GX22, PPV6/GX11, PPV6/GX51) were 6112 nucleotides (nt) in length and four isolates (PPV6/GX01, PPV6/GX12, PPV6/GX18, PPV6/GX44) were $6111 \mathrm{nt}$ in length, which lacks one base (T) in the 3'UTR compared to the other 6 strains. The ORF1 encodes a putative NS1 of 663 amino acids and ORF2 encodes a putative VP1 of 1190 amino acids. Of note, in this study, in the 3' UTRs, 4 of the 10 isolates lacked 15 nucleotides and the remaining 6 isolates lacked 14 amino acids (Fig. 2), which resulted in significant changes in RNA secondary structure (Supplementary Fig. 1).

Based on nucleotide similarity analysis of the complete coding region, the 10 sequences shared 98.9\%-99.6\% similarity, with $99.4 \%-100 \%$ similarity in the NS1 gene and $98.4 \%-99.2 \%$ similarity in the VP1 gene. In addition, ten PPV6 sequences shared $99.3 \%-99.4 \%$ similarity in NS1 gene and $96.0 \%-97.1 \%$ similarity in the VP1 gene compared with reference strains. Comparisons of the genome sequences of the PPV6 strains clade and with its sister clade containing PPV4 and PPV5 found $41.6 \%-48.8 \%$ identity with PPV4 and 41.5\%-42.1\% identity with PPV5. Within the VP1 unique region, compared with PPV1, PPV2 and PPV3, the motifs of the $\mathrm{Ca}^{2+}$ binding loop (YTGPR) and the catalytic residues (HDIRY) of putative secretory PLA2 were very conserved without amino acid mutation (Fig. 3).

\section{Phylogenetic analysis}

In order to better understand the genetic relationship between the strains identified in this study, a phylogenetic tree was constructed using the neighbor joining $(\mathrm{NJ})$ method. Based on amino acid sequences of the NS1 protein which commonly used to differentiate members of the subfamily Parvovirinae, all PPV6 strains identified in this study were closely related to PPV6 reference strains and located in the genus Copiparvovirus together with PPV4 and PPV5 (Fig. 4a).

To determine the genetic relationship of PPV6 isolates to other PPV6 reference strains downloaded from GenBank. A phylogenetic tree was constructed using the maximum likelihood method comparing the VP1 amino acid sequences of PPV6 (Fig. 4b). The results showed that PPV6 strains clustered in two distinct groups (A and B). Interestingly, we found that almost all PPV6 strains isolated from China clustered in group B, except for the three Chinese strains (AH-PPV620178-1, TJ and FJ2017) that clustered in group A. 
Compare with reference strains in group B, ten PPV6 sequences shared 97.6\%-99.6\% similarity in the VP1 gene. Especially, PPV6/GX05 strain shared 99.6\% homology (5 amino acid difference) with BJ2 strain (KF999682, China/2012). PPV6/GX18 strain shared 99.1\% homology with (11 amino acid difference) AHPPV620178-2 strain (MW853956, 2017/China).

Further we analyze the relationship between the evolutionary tree branching and amino acid substitution

of PPV6. By combining the results of multiple sequence alignment and genetic evolution, in this study, a total of 14 amino acid mutation were found, namely R14K/Q, I116L/K/T, N788S, R789/M, S/T791H, M792H, A815S, D873G, N919S, R938K, S941T, R1013H, T1038N, T1042D/Y, which result in the evolutionary tree were divided into two distinct branches (A, B) (Fig. 4c).

\section{Amino acid mutation analysis of the isolates}

Base substitution and amino acid mutation analyses were performed by comparison with the PPV6 reference strain which from difference geographic area (Supplementary Table S3). A previous study indicated that there may be three conserved regions in the PPV6 VP1 protein including two regions of greater than 100 amino acids in length that are conserved (414-787 and 1090-C-terminus) and a slightly smaller conserved stretch from 164-256 (Fig. 5a). In this study, compared with difference PPV6 sequences identified in different geographic regions, we found that the VP1 gene is more prone to mutation than the NS1 gene. In the NS1, two adaptive mutations $(\mathrm{Q} 3 \mathrm{H}, \mathrm{P} 2 \mathrm{OH})$ appear in most isolates. Meanwhile, in the VP1, all the three conserved regions have amino acid mutations (Fig. 5b). In the first conserved region (aa 164-256), only one amino acid mutation was observed (R217H), was present in PPV6/GX18. In the second conserved region (aa 414-787), 20 amino acid mutations were observed that is not found in PPV6 strains sequenced from any other regions. From which, PPV6/GX01 strain contain 5 amino acid mutations (A619T, C724R, P752L, P770L, P774H). PPV6/GX05 strain contain 2 amino acid mutations (C602F, D702E). PPV6/GX11 strain contain 1 amino acid mutations (T731M). PPV6/GX12 strain contain 5 amino acid mutations (R451H, T547S, A619T, Q638H, Q730E). PPV6/GX18 strain contain 3 amino acid mutations (A619T, T626P, D702E). PPV6/GX22 strain contain 1 amino acid mutations (A619T). PPV6/GX33 strain contain 3 amino acid mutations (V522A, A619T, A666P). PPV6/GX41 strain contain 4 amino acid mutations (R451H, T547S, C602F, Q730E). PPV6/GX44 strain contain 6 amino acid mutations (D471V, L501F, D561G, C601R, P658H, Q730E). PPV6/GX51 strain contain 2 amino acid mutations (A619T, Q730E). The third region (aa 1090-1190) only one amino acid mutation was observed (F1120I), was present in PPV6/GX05. Interestingly, we also found that 2 adaptive mutations (A619T, Q730E) appear in most isolates. This mutation may prove useful in the future for tracking the prevalence of different PPV6 strains. At present, the relationship between PPV6 pathogenicity, tissue tropism and VP1 amino acid variation has not been determined.

Besides, we analyzed the possible B-lymphocyte and T-lymphocyte epitopes on the NS1 gene and VP1 gene, respectively. In this study, in the NS1, amino acids at positions 534-554, 512-525, 182-194, 575585, 605-615, 225-233, 641-649 may exist B lymphocyte antigenic epitopes (Supplementary Fig. 2-a); amino acids at positions 333-341, 395-403 may exist T lymphocytes CTL epitopes (Supplementary Fig. 2-b). In the VP1, amino acids at positions 926-941, 790-805, 1098-1113, 525-540, 345-360 may 
exist B lymphocyte antigenic epitopes (Supplementary Fig. 2-c); amino acids at positions 207-216, 501510, 765-774 may exist T lymphocytes CTL epitopes (Supplementary Fig. 2-d). Most of these possible antigenic sites are located in the mutated positions of the conserved regions of the VP1 gene. The analysis results provide new research ideas for the study of epitope vaccines.

\section{Discussion}

Porcine parvovirus is one of the major causative agents of reproductive failure syndromes in pigs and is characterized by miscarriage, infertility, mummified fetuses and stillbirths [6]. PPV1 was first discovered in Germany in 1965. Subsequently, PPV1 was detected in China in 1983, which caused great economic losses to the pig industry in China. In recent years, with the development of molecular biology and the use of pathogen discovery tools, a number of novel porcine parvoviruses have been identified all over the world, including PPV2 PPPV7 [17]. PPV6 was first detected by Ni et al [1] in aborted pig fetuses in 2012. In addition, the study showed that the infection rate of PPV 6 in aborted pig fetuses and piglets $(50 \%$ and $75 \%$, respectively) was significantly higher than that of fattening pigs and sows $(15.6 \%$ and $3.8 \%$, respectively).

Parvovirus was reported to be co-infected with multiple viruses, causing more severe disease. For example, co-infection of equine parvovirus-hepatitis (EqPV-H) and equine hepatitis virus (EqHV) causes fulminant equine hepatitis [18]; co-infection of goose parvovirus-associated virus with duck circovirus causes feather shedding syndrome (FSS) [19]; co-infection of Parvovirus B19 with influenza A/H1N1 causes fulminant myocarditis and pneumonia [20]. In the most pig-producing country, a high level of PPV and PCV co-infection is common. The prevalence of PPV1 ranged from 25.8\%-71.9\% $[15,21]$. Previous report that PPV1 can co-infection with PCV2, PCV3, porcine reproductive and respiratory syndrome virus (PRRSV), pseudorabies virus (PRV), and classical swine fever virus (CSFV) [22]. The prevalence of PP1 and PCV2 co-infection is high, and PPV1 may trigger PCV2 associated disease (PCVAD) by supporting PCV2 replication, and increase porcine circovirus-associated disease (PCVAD) severity [23]. It is reported that the positive rate of PPV7 in PCV2 positive pig farms was significantly higher than that in negative farms (65.5\% and 5.7\%, respectively) [24]. PPV6 was also reported to be co-infection with PCV and associated with the reproduction system of the sow [13]. Previous report studied that in PCV3 positive samples, PCV3 had a high co-infection rate with both PPV6 (60.0\%) and PPV7 (74.3\%) [25]. Based on these data, we inferred that there is a possible association between PP6 and PCV. In this study, PPV6 also had a high co-infection rate with PCV (69.4\%). Meanwhile, we found that the positive rate of PPV6 coinfection with PCV was higher than that of PPV6 infection alone (30.6\%). The positive rate of PPV6 coinfected with PCV2 was significantly higher than that of PPV6 co-infection with PCV3 and PCV4. The results inferred that PPV6 is likely a significant co-factor in PCVAD. Although the role of PPVs as a causal agent for PCVAD has not yet been unveiled, swine are important for generating genetic diversity for parvoviruses [26]. Moreover, whether PPV6 is mutated to produce variants with epidemiological consequences needs further research to confirm. 
The PPV6 genome is approximately $6 \mathrm{~K}$ nt in length and contains two open reading frames (ORFs), $5^{\prime}$ and 3 ' non-coding regions. The nonstructural protein (NS1) encoded by ORF1 and the capsid protein (VP1) encoded by ORF2 play important roles in viral replication and cell infection, respectively [1]. Previous studies have shown that the parvovirus $\mathrm{H}-1$ NS2 protein affects viral gene expression through sequences in the 3' untranslated region [27]. In this study, four isolates were 6111 nucleotides (nt) in length and six isolates were $6112 \mathrm{nt}$ in length. In addition, we found that in the $3^{\prime}$ UTRs, 4 of the 10 isolates lacked 15 nucleotides and the remaining 6 isolates lacked 14 amino acids, which resulted in significant changes in RNA secondary structure. Interestingly, we found the same base mutation when compared with the PPV6 strain (BJ2) first discovered in China in 2012. The effect of the deletion of bases in the 3'UTR on the pathogenicity of PPV6 in this study needs to be further confirmed.

The presence of the calcium $\left(\mathrm{Ca}^{2+}\right)$ binding loop and the phospholipase A2 domain (PLA2), features found in many other parvoviruses, was identified in ORF2. The amino acid sequence of the $\mathrm{Ca}^{2+}$ binding loop was "YXGXG" in PPV1, PPV2, PPV3 and PPV5, and was absent in PPV4 [6, 28]. The amin acid sequence of the $\mathrm{Ca}^{2+}$ binding loop was "YXGXXG" in PPV7 [6]. The PLA2 were found to be conserved among PPV6 strains. However, PLA2 are absent in PPV7. In this study, we found that in VP1, compared with PPV1, PPV2 and PPV3, the motifs of the $\mathrm{Ca}^{2+}$ binding loop (YTGPR) and the catalytic residues (HDIRY) of putative secretory PLA2 were very conserved without amino acid mutation, which is consistent with the results of other researchers $[1,10]$, namely the 'YTGPR' motif and the 'HDIRY' motif, respectively.

The NS1 protein which is commonly used to differentiate members of the subfamily Parvovirinae. In this study, all PPV6 strains identified in Guangxi province were closely related to PPV6 reference strains and located in the genus Copiparvovirus together with PPV4 and PPV5. The genetic evolutionary tree of VP1 was divided into two main branches $(A, B)$, which shared most mutation in the non-conserved region. Interestingly, most of the isolates from China clustered in group $B$, while those isolated from different geographic regions (United States, Poland, Korea, Canada, Spain, Brazil, etc.) clustered in group A. Therefore, we speculate that the geographical isolation of the PPV6 epidemic in China leads to the emergence of different branches from other countries.

Among all strains identified in different geographic regions (North America, China, Poland), VP1 resembled variable proteins in the PPV6 genome and was the most diverse compared to NS1. This conforms to the predicted behavior of the virus in response to the host immune system, in that the capsid protein would be under the greatest direct pressure from the host immune system. This view has been confirmed by many research $[1,10,17]$. The importance of these amino acids substitutions is currently unknown though amino acids changes of PPV VP1 are reported to be responsible for the pathogenicity of the PPV strains [17]. Multi-epitope vaccine is a new type of vaccine developed in recent years, which is constructed by integrating multiple B cells and T cells on the viral protein into a specific vector [29]. They have unique advantages in inhibiting viral replication and fighting immune escape strains [30, 31]. In the process of epitope vaccine research, the screening of viral protein B cell epitopes and CTL epitopes in $T$ cells is particularly important. The selection of CTL epitopes plays an important role in clearing viral 
infection and providing immune protection [32]. In this study, compare with the reference strain VP1, these isolates of PPV6 have 22 amino acid mutation in conversed region, which had never been reported. The VP1 gene is more prone to mutation than NS1 gene. Interestingly, two adaptive mutations were found in NS1 (Q3H, P2OH) and VP1 (A619T, Q730E), respectively. We speculate that, over time, new strains of dominant strains will emerge that are adapted to the environment. Based on these data, we inferred that this mutation may prove useful in the future for tracking the prevalence of different PPV6 strains. In addition, the relationship between PPV6 pathogenicity and VP1 amino acid variation need further, study. Compared with the VP1 protein, the NS1 protein structure of the PPV6 isolates is more stable, and the predicted dominant antigenic epitopes will provide a direction for the research and creation of future PPV6 epitope vaccines.

Our results offer novel insights into the prevalence of porcine parvoviruses 6 in Guangxi, China and its coinfection with porcine circovirus. At the same time, it provides new research ideas for epitope vaccine research. With the continuous evolution and mutation of PPV6, effective vaccines should be researched as soon as possible and prevention and control measures should be strengthened to prevent irreversible losses to the pig industry.

\section{Conclusion}

In this study, we investigated the prevalence of PPV6 in Guangxi province and conducted genome sequencing of the PP6 strains found in this province. There is a high prevalence of PPV6 and PCV coinfection in PPV6-positive pigs. The effect of the deletion of bases in the 3' UTR on the pathogenicity of PPV6 in this study needs to be further confirmed. Notably, compared with the VP1 protein, the NS1 protein structure of the PPV6 isolates is more stable, and the predicted dominant antigenic epitopes will provide a direction for the research and creation of future PPV6 epitope vaccines.

\section{Abbreviations}

A: alanine; C: cystine; CTL: cytotoxic lymphocyte D: aspartic acid; E: glutamic acid; F: phenylalanine; $\mathbf{G}$ : glycine; $\mathbf{H}$ : histidine; I: isoleucine; $\mathbf{K}$ : lysine; L: leucine; M: methionine; $\mathbf{M L}$ : Maximum likelihood; $\mathbf{N}$ : asparagine; NJ: Neighbor joining; NSP: non-structural protein; ORF: open-reading frame; PPV: porcine parvovirus; PCV: porcine circovirus; PMWS: post-weaning multi-systemic wasting syndrome; PCVAD: PCVassociated disease; PDNS: porcine dermatitis and nephropathy syndrome; P: proline; Q: glutamine; R: arginine; S: serine; SMEDI: Stillbirths, mummification, embryonic death and infertility; T: threonine; VP1: capsid protein; V:valine; Y: tyrosine.

\section{Declarations}

Acknowledgements 
We would like to thank Ningning Hu and Jin Zhao for their assistance with reagent preparation. And we would like to thank Wei Wang for editing the manuscript.

\section{Authors' contributions}

NY and ZPY performed the research, data analysis and wrote the manuscript. LEC helped in the writing of the manuscript. YML, SF, ZXL, ZXY, CHL, ZXY and $\mathrm{HZ}$ participated in sample collection and research testing. WW, XYZ, WCS, and NYJ designed the research. All authors read and approved the final manuscript.

\section{Funding}

This work was supported by the following Grants: National Key Research and Development Program of China (Grant numbers 2018YFD0500104). The funding body was solely involved in funding and had no role in the design of the study, the collection, analysis, and interpretation of the data, nor in writing the manuscript.

\section{Availability of data and materials}

The datasets generated and/or analysed during the current study are available in the NCBI repository (Accession number: OM811448-0M811457).

\section{Ethics approval and consent to participate}

Pig serum samples used in this study were obtained from the Guangxi Center for Animal Disease Control and Prevention and the experiment was approved by Guangxi University Animal Welfare and the Animal Experimental Ethical Committee, approval number NO. Xidakezi2000138. Written informed consent for the use of samples before participation in the study was obtained from the pig' owner. All methods were carried out in accordance with relevant guidelines and regulations. All methods are reported in accordance with ARRIVE guidelines for the reporting of animal experiments. No agents were administered to pigs in any way in this study.

\section{Consent to publish}

Not applicable.

\section{Competing interests}

The authors declare that they have no competing interests.

\section{References}

1. Ni J, Qiao C, Han X, et al. Identification and genomic characterization of a novel porcine parvovirus (PPV6) in China.Virology journal,2014.11: p.203. 
2. Liu P, Chen $S$, Wang M, et al., The role of nuclear localization signal in parvovirus life cycle. Virology journal,2017.14(1):p.80.

3. Cotmore SF, Agbandje-McKenna M, Chiorini JA, et al., The family Parvoviridae. Archives of virology,2014.159(5): p.1239-47.

4. Glasa M, Palkovics L, Komínek P., et al., Geographically and temporally distant natural recombinant isolates of Plum pox virus (PPV) are genetically very similar and form a unique PPV subgroup. The Journal of general virology,2004. 85:p.2671-2681.

5. Oh W, Kim RY, Nguyen VG, et al., Perspectives on the Evolution of Porcine Parvovirus. Viruses, 2017.9(8).

6. Wang W. Cao L. Sun W, et al. Sequence and phylogenetic analysis of novel porcine parvovirus 7 isolates from pigs in Guangxi, China. PloS one,2019.14(7): p.e0219560.

7. Johnson R. and D Collings. Experimental infection of piglets and pregnant gilts with a parvovirus. The Veterinary record,1969.85(16): p.446-7.

8. Mészáros I., Olasz F, Cságola A, et al. Biology of Porcine Parvovirus (Ungulate parvovirus 1). Viruses,2017.9(12).

9. Bolt DM, Häni H, Müller E, et al.Non-suppurative myocarditis in piglets associated with porcine parvovirus infection. Journal of comparative pathology,1997.117(2): p.107 - 18.

10. Cui J, Fan J, Gerber PF, et, al.,First identification of porcine parvovirus 6 in Poland. Virus genes, 2017. 53(1): p.100-104.

11. Palinski RM, Mitra N and Hause BM. Discovery of a novel Parvovirinae virus, porcine parvovirus 7 , by metagenomic sequencing of porcine rectal swabs. Virus genes, 2016.52(4): p.564-7.

12. Opriessnig T, Xiao CT, Gerber PF, et al. Identification of recently described porcine parvoviruses in archived North American samples from 1996 and association with porcine circovirus associated disease.Veterinary microbiology,2014.173: p.9-16.

13. Opriessnig, $T$ and Halbur, $P G$. Concurrent infections are important for expression of porcine circovirus associated disease. Virus research, 2012. 164: p. 20-32.

14. Sun J, Huang L, Wei Y, et al.Prevalence of emerging porcine parvoviruses and their co-infections with porcine circovirus type 2 in China. Archives of virology.2015.160(5):p.1339-44.

15. Cságola, A, Lőrincz, M, Cadar, D, et al., Detection, prevalence and analysis of emerging porcine parvovirus infections. Archives of virology, 2012. 157(6): p. 1003-10.

16. Allan GM, Kennedy S, McNeilly F, et al. Experimental reproduction of severe wasting disease by coinfection of pigs with porcine circovirus and porcine parvovirus. Journal of comparative pathology,1999.121(1): p.1-11.

17. Schirtzinger EE, Suddith AW, Hause BM, et al. First identification of porcine parvovirus 6 in North America by viral metagenomic sequencing of serum from pigs infected with porcine reproductive and respiratory syndrome virus. Virology journal,2015.12: p.170. 
18. Yoon, J, Park, T, Kim, A, et al. First report of equine parvovirus-hepatitis and equine hepacivirus coinfection in horses in Korea. Transboundary and emerging diseases, 2021.

19. Yang, $Y$, Sui, N, Zhang, R, et al. Coinfection of novel goose parvovirus-associated virus and duck circovirus in feather sacs of Cherry Valley ducks with feather shedding syndrome.2020,99(9): p.4227-4234.

20. Callon, D, Berri, F, Lebreil, AL, et al. Coinfection of Parvovirus B19 with Influenza A/H1N1 Causes Fulminant Myocarditis and Pneumonia. An Autopsy Case Report. Pathogens.2021,10(8).

21. Saekhow, P, Kishizuka, S, Sano, N, et al. Coincidental detection of genomes of porcine parvoviruses and porcine circovirus type 2 infecting pigs in Japan. The Journal of veterinary medical science. 2016,77(12),p:1581-1586

22. Mai, J, Wang, D, Zou, Y, et al. High Co-infection Status of Novel Porcine Parvovirus 7 With Porcine Circovirus 3 in Sows That Experienced Reproductive Failure. Frontiers in veterinary science.2021.8:p. 695553.

23. Opriessnig, T, Fenaux, M, Yu, S, et al. Effect of porcine parvovirus vaccination on the development of PMWS in segregated early weaned pigs coinfected with type 2 porcine circovirus and porcine parvovirus. Veterinary microbiology.2004,98, p: $209-20$.

24. Xing, $X$, Zhou, $H$, Tong, L, First identification of porcine parvovirus 7 in China. Archives of virology.2018. 163(1).p:209-213.

25. $\mathrm{Ha}, \mathrm{Z}, \mathrm{Xie}, \mathrm{CZ}, \mathrm{Li}, \mathrm{JF}$, Molecular detection and genomic characterization of porcine circovirus 3 in pigs from Northeast China. BMC veterinary research.2018. 14(1).p:321

26. Ramamoorthy, $S$, Meng, XJ. Porcine circoviruses: a minuscule yet mammoth paradox. Animal health research reviews, 2019.1(10):p1-20

27. Li, X., Rhode, S. L., 3rd. The parvovirus H-1 NS2 protein affects viral gene expression through sequences in the $3^{\prime}$ untranslated region. Virology,1993.194(1):10-19

28. Xiao, CT, Giménez-Lirola, LG, Jiang YH, et, al. Characterization of a novel porcine parvovirus tentatively designated PPV5. PloS one,2013.8(6):P e65312

29. Zhang, L. Multi-epitope vaccines: a promising strategy against tumors and viral infections. Cellular \& molecular immunology.2018.15(2).P:182-184

30. Xiao, Y, Lu, Y and Chen, YH. Epitope-vaccine as a new strategy against HIV-1 mutation. Immunology letters.2001.77(1).p:3-6

31. Raymond, DD, Bajic, G, Ferdman, J, et al. Conserved epitope on influenza-virus hemagglutinin head defined by a vaccine-induced antibody. Proceedings of the National Academy of Sciences of the United States of America. 2018.115(1).p:168-173

32. Kumar Pandey, R, Ojha, R, Mishra, A, et al. Designing B- and T-cell multi-epitope based subunit vaccine using immunoinformatics approach to control Zika virus infection. Journal of cellular biochemistry. 2018.119(9). P:7631-7642. 
Figures

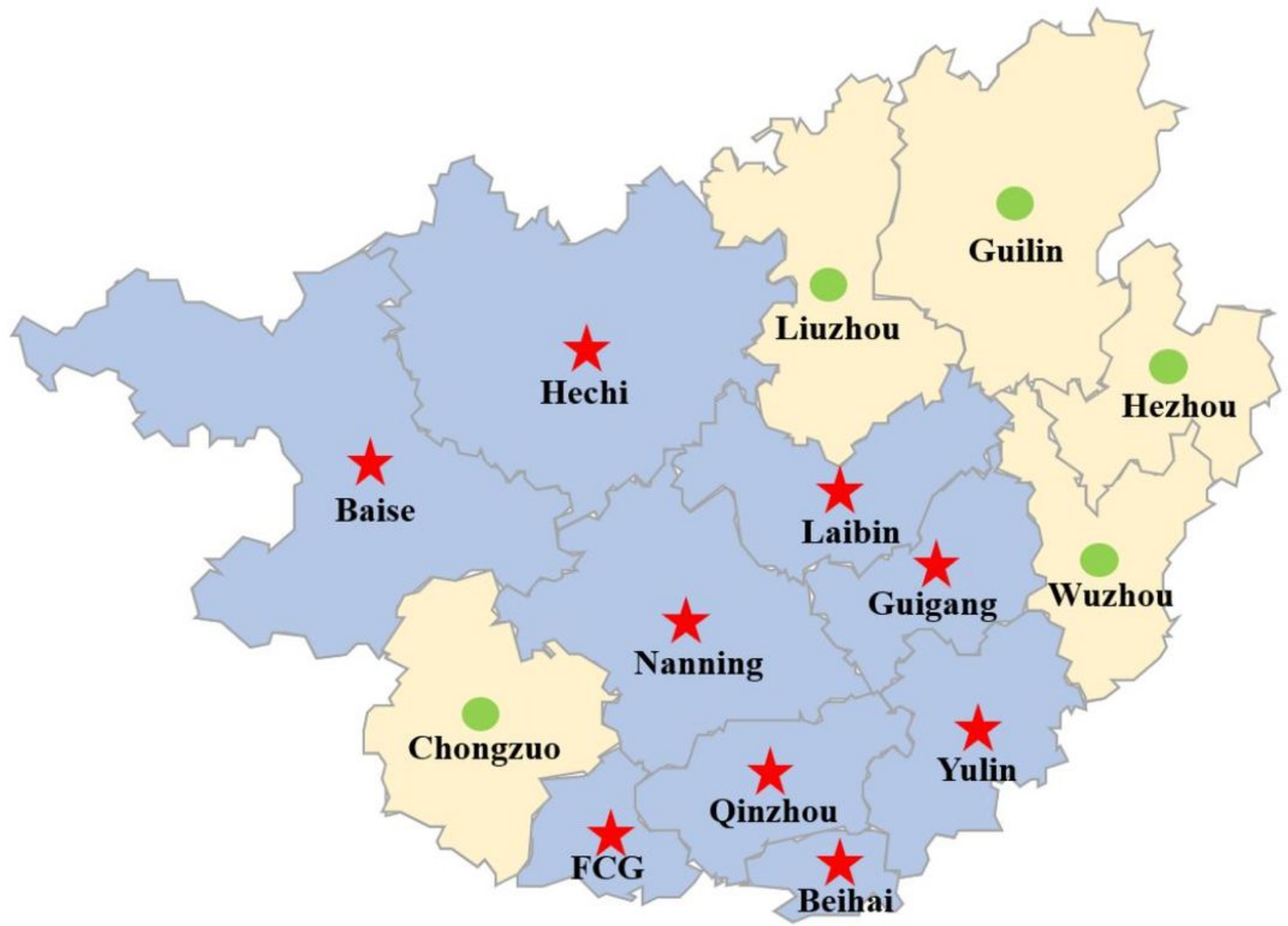

Figure 1

Geographical information for serum samples in Guangxi, China. Red stars indicate the geographical location of the sample. 


\begin{tabular}{|c|c|c|}
\hline & $5920------$ & 6030 \\
\hline PPV6/GX01 & T A A $\ldots \ldots \ldots$ & T C T T T $\ldots$ T T T GA T \\
\hline$P P V 6 / G X 18$ & T A $\mathbf{A} \ldots \ldots$ T T C & T C T T T $\ldots$ T T T G A T \\
\hline BJ2_China:Beijing & T A A $\ldots \ldots \ldots$ T T & - T T T G A T \\
\hline$P P \bar{V} 6 / G X 44$ & T A A $\ldots \ldots \ldots$ T T & T C T T T $\ldots$ T T T GA T \\
\hline$P P V 6 / G X 11$ & T A A $\ldots \ldots \ldots$ T T C & T C T T IT T - T T T G A T \\
\hline PPV6/GX51 & T A A $\ldots \ldots \ldots$ T T C & T C T T T T - T T T GA T \\
\hline$P P V 6 / G X 33$ & $\mathrm{TAA} \ldots \ldots \mathrm{T} \mathrm{T} C$ & T C T T T T \\
\hline PPV6 & T A $\mathbf{A} \ldots \ldots-\ldots \mathrm{T}$ T & T C T T T T T - T T T G A T \\
\hline PPV6 & T A A $\ldots \ldots$ T T C & T C T T T - - T T T GA T \\
\hline$P P V C$ & T A A $\ldots \ldots \ldots$ T $\mathbf{C}$ & T C T T T T - T T T G A T \\
\hline$P P V 6 / G X 41$ & T A A $\ldots \ldots \ldots$ T T C & T C T T T T - T T T GA T \\
\hline Br_Brazil & TA A T T C G T G T T G T A A T T C & T C T T T T - T T T GA T \\
\hline$\overline{S U} 6-I A-2014 \_U S A$ & TA A T T C G C G C T G T G A T T C & T C T T T T - T T T G A T \\
\hline KSU3-KS-2014_USA & TA T T C G T G T T G T GATT C & T C T T T \\
\hline KSU1-AZ-2014_USA & TA A T T C G T G T T G T A T T C & T C T T T $\ldots$ T T T G A T \\
\hline TJ_China:Fujian & TAAT T C G T G T T G T GA T T C & T C T T T $\ldots$ T T T GA T \\
\hline$K \overline{S U} 2-A Z-2014 \_U S A$ & T A A T T C G T G T T G T G A T C & T C T T T $\ldots$ T T T G A T \\
\hline FJ2017_China:Beijing & T A A T T C G T G T T G T G A T T C & T C T T T $\ldots$ T T T GA T \\
\hline SK-CA-2018_Canada & T A A T T C G T G T T G T A A T T C & T C T T T T T T T T T G A T \\
\hline 3456/2012_Spain & T A A T T C G T G T T G T A A T T C & T C C T T T _ T T T G A T \\
\hline D-2014_USA & TA A T T C G T G T T G T A A T T C & T C T T T T T - T T T G A T \\
\hline KSU4-NE-2014_USA & 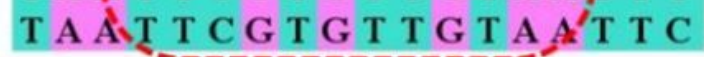 & T C T T T T -- T T T GA T \\
\hline
\end{tabular}

Figure 2

Alignment of nucleotide acid sequences of nucleotide acids representing different isolates of PPV6. The red box highlights the sequence region where missing nucleotides were identified. 


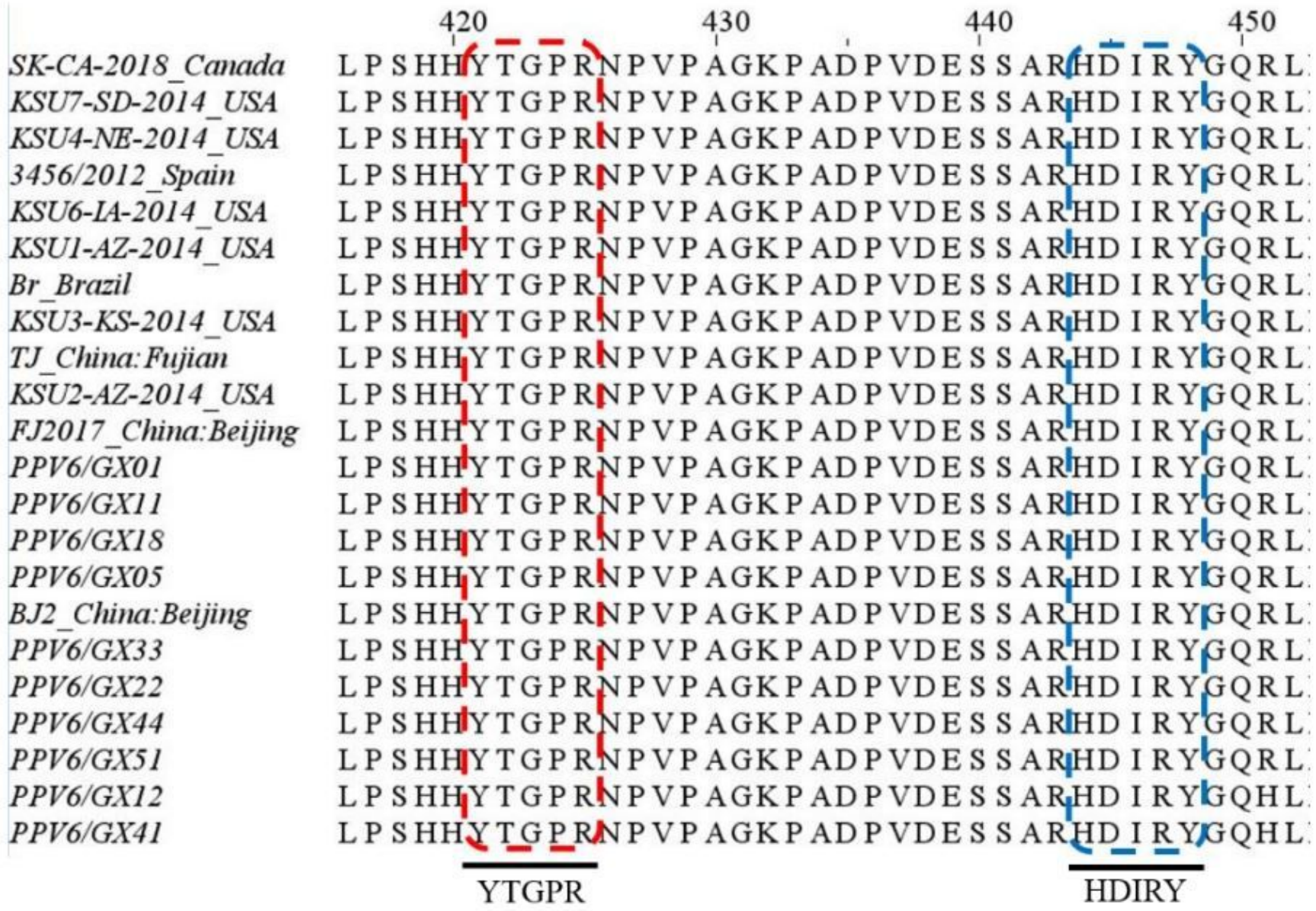

Figure 3

Sequence alignment of the putative phospholipase A2 motif of PPV6 with reference parvoviruses strains. The conserved amino acids of the $\mathrm{Ca}^{2+}$ binding loop (YTGPR) and the catalytic residues (HDIRY) are indicated at the bottom of the alignment. The red and blue box are highlight the conserved amino acids of the $\mathrm{Ca}^{2+}$ binding loop and catalytic residues, respectively. 

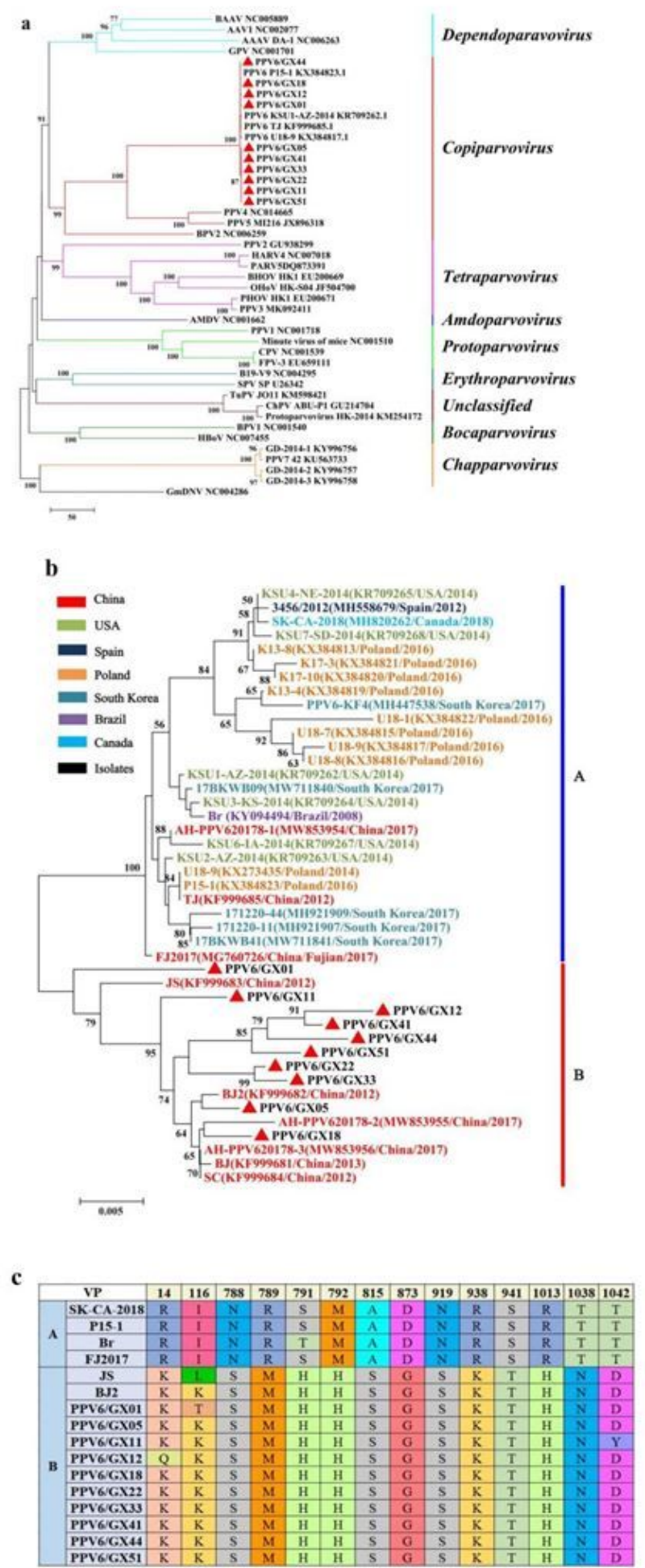

\section{Figure 4}

Phylogenetic analysis of PPV6 isolates. The strains identified in this study are represented with red circles (A). a. Phylogenetic relationship of PPV6 with subfamily Parvovirinae. The phylogenetic tree was inferred from amino acid sequences of NS1 by the NJ method with 1000 bootstrap resampling. $\mathbf{b}$. The phylogenetic tree of the amino acid sequence of PPV6 VP1 was constructed by ML method. Bootstrap 
values (1000 replicates) < 50\% are not show. c. Mutations that cause branches in the genetic tree. VP1 Amino acid mutations in non-conserved region resulting in distinct group $(A, B)$.

$\mathbf{a}$

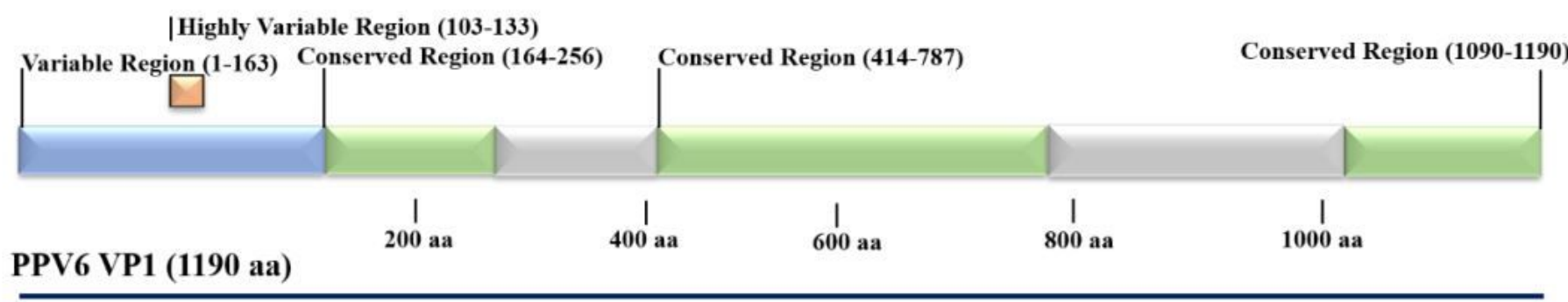

b

\begin{tabular}{|c|c|c|c|c|c|c|c|c|c|c|c|c|c|c|c|c|c|c|c|c|c|c|c|c|c|c|}
\hline & \multicolumn{4}{|c|}{ NS1 } & \multicolumn{22}{|c|}{ VP1 } \\
\hline Position & 3 & 8 & 20 & 28 & 217 & 451 & 471 & 501 & 522 & 547 & 561 & 601 & 602 & 619 & 626 & 638 & 658 & 666 & 702 & 724 & 730 & 731 & 752 & 770 & 774 & 1120 \\
\hline BJ2 & Q & $\mathrm{G}$ & $\mathrm{P}$ & I & $\mathrm{R}$ & $\mathrm{R}$ & $\mathrm{D}$ & $\mathrm{L}$ & $\mathrm{V}$ & $\mathrm{S}$ & $\mathrm{D}$ & $\mathrm{C}$ & $\mathrm{C}$ & A & $\mathrm{T}$ & Q & $\mathrm{P}$ & $\mathrm{A}$ & $\mathrm{D}$ & $\mathrm{C}$ & $\mathrm{Q}$ & $\mathrm{T}$ & $\mathrm{P}$ & $\mathrm{P}$ & $\mathrm{P}$ & $\mathrm{F}$ \\
\hline KSU2-AZ-2014 & $Q$ & $\mathrm{G}$ & $\mathrm{P}$ & $\mathrm{I}$ & $\mathrm{R}$ & $\mathrm{R}$ & $\mathrm{D}$ & $\mathrm{L}$ & $\mathrm{V}$ & $\mathrm{S}$ & $\mathrm{D}$ & $\mathrm{C}$ & $\mathrm{C}$ & $\mathrm{A}$ & $\mathrm{T}$ & $\mathrm{Q}$ & $\mathrm{P}$ & A & $\mathrm{D}$ & $\mathrm{C}$ & $\mathrm{Q}$ & $\mathrm{T}$ & $\mathrm{P}$ & $\mathrm{P}$ & $\mathrm{P}$ & $\mathrm{F}$ \\
\hline P15-1 & Q & G & $\mathrm{P}$ & I & $\mathrm{R}$ & $\mathrm{R}$ & $\mathrm{D}$ & $\mathrm{L}$ & $\mathrm{V}$ & $\mathrm{S}$ & $\mathrm{D}$ & $\mathrm{C}$ & $\mathrm{C}$ & $\mathrm{A}$ & $\mathrm{T}$ & $\mathrm{Q}$ & $\mathrm{P}$ & $\mathrm{A}$ & $\mathrm{D}$ & $\mathrm{C}$ & $\mathrm{Q}$ & $\mathrm{T}$ & $\mathrm{P}$ & $\mathrm{P}$ & $\mathrm{P}$ & $\mathrm{F}$ \\
\hline 17BKWB09 & Q & $\mathrm{G}$ & $\mathrm{P}$ & I & $\mathrm{R}$ & $\mathrm{R}$ & $\mathrm{D}$ & $\mathrm{L}$ & $\mathrm{V}$ & $\mathrm{S}$ & $\mathrm{D}$ & $\mathrm{C}$ & $\mathrm{C}$ & $\mathrm{A}$ & $\mathrm{T}$ & $\mathrm{Q}$ & $P$ & $\mathrm{~A}$ & $\mathrm{D}$ & $\mathrm{C}$ & $\mathrm{Q}$ & $\mathrm{T}$ & $\mathrm{P}$ & $\mathrm{P}$ & $\mathrm{P}$ & $\mathrm{F}$ \\
\hline SK-CA-2018 & $Q$ & $\mathrm{G}$ & $\mathrm{P}$ & $\mathrm{I}$ & $\mathrm{R}$ & $\mathrm{R}$ & $\mathrm{D}$ & $\mathrm{L}$ & $\mathrm{V}$ & $\mathrm{S}$ & $\mathrm{D}$ & $\mathrm{C}$ & $\mathrm{C}$ & $\mathrm{A}$ & $\mathrm{T}$ & $Q$ & $\mathrm{P}$ & $\mathrm{A}$ & $\mathrm{D}$ & $\mathrm{C}$ & $\mathrm{Q}$ & $\mathrm{T}$ & $\mathrm{P}$ & $\mathrm{P}$ & $\mathrm{P}$ & $\mathrm{F}$ \\
\hline $3456 / 2012$ & $Q$ & $\mathrm{G}$ & $\mathrm{P}$ & $\mathrm{I}$ & $\mathrm{R}$ & $\mathrm{R}$ & $\mathrm{D}$ & $\mathrm{L}$ & $\mathrm{V}$ & $\mathrm{S}$ & $\mathrm{D}$ & $\mathrm{C}$ & $\mathrm{C}$ & $\mathrm{A}$ & $\mathrm{T}$ & $Q$ & $\mathrm{P}$ & $\mathrm{A}$ & $\mathrm{D}$ & $\mathrm{C}$ & $\mathrm{Q}$ & $\mathrm{T}$ & $\mathrm{P}$ & $\mathrm{P}$ & $\mathrm{P}$ & $\mathrm{F}$ \\
\hline $\mathrm{Br}$ & Q & $\mathrm{G}$ & $\mathrm{P}$ & I & $\mathrm{R}$ & $\mathrm{R}$ & $\mathrm{D}$ & $\mathrm{L}$ & $\mathrm{V}$ & $\mathrm{S}$ & D & $\mathrm{C}$ & $\mathrm{C}$ & $\mathrm{A}$ & $\mathrm{T}$ & Q & $\mathrm{P}$ & A & $\mathrm{D}$ & $\mathrm{C}$ & $\mathrm{Q}$ & $\mathrm{T}$ & $\mathrm{P}$ & $\mathrm{P}$ & $\mathrm{P}$ & $\mathrm{F}$ \\
\hline PPV6/GX01 & Q & $\mathrm{G}$ & $\mathrm{P}$ & $\mathrm{M}$ & $\mathrm{R}$ & $\mathrm{R}$ & $\mathrm{D}$ & $\mathrm{L}$ & $\mathrm{V}$ & $\mathrm{S}$ & $\mathrm{D}$ & $\mathrm{C}$ & $\mathrm{C}$ & $\mathrm{T}$ & $\mathrm{T}$ & Q & $\mathrm{P}$ & $\mathrm{A}$ & $\mathrm{D}$ & $\mathrm{R}$ & $\mathrm{Q}$ & $\mathrm{T}$ & $\mathrm{L}$ & $\mathrm{L}$ & $\mathrm{H}$ & $\mathrm{F}$ \\
\hline PPV6/GX05 & $\mathrm{H}$ & $\mathrm{G}$ & $\mathrm{H}$ & I & $\mathrm{R}$ & $\mathrm{R}$ & $\mathrm{D}$ & $\mathrm{L}$ & $\mathrm{V}$ & $\mathrm{S}$ & $\mathrm{D}$ & $\mathrm{C}$ & $\mathrm{F}$ & $\mathrm{A}$ & $\mathrm{T}$ & $\mathrm{Q}$ & $\mathrm{P}$ & $\mathrm{A}$ & $\mathrm{E}$ & $\mathrm{C}$ & $\mathrm{Q}$ & $\mathrm{T}$ & $\mathrm{P}$ & $\mathrm{P}$ & $\mathrm{P}$ & I \\
\hline PPV6/GX11 & $\mathrm{H}$ & G & $\mathrm{H}$ & $\mathrm{I}$ & $\mathrm{R}$ & $\mathrm{R}$ & $\mathrm{D}$ & $\mathrm{L}$ & $\mathrm{V}$ & $\mathrm{S}$ & $\mathrm{D}$ & $\mathrm{C}$ & $\mathrm{C}$ & $\mathrm{A}$ & $\mathrm{T}$ & $\mathrm{Q}$ & $\mathrm{P}$ & A & $\mathrm{D}$ & $\mathrm{C}$ & $\mathrm{Q}$ & $\mathrm{M}$ & $\mathrm{P}$ & $\mathrm{P}$ & $\mathrm{P}$ & $\mathrm{F}$ \\
\hline PPV6/GX12 & Q & $\mathrm{D}$ & $\mathrm{P}$ & I & $\mathrm{R}$ & $\mathrm{H}$ & $\mathrm{D}$ & $\mathrm{L}$ & $\mathrm{V}$ & $\mathrm{T}$ & D & $\mathrm{C}$ & $\mathrm{C}$ & $\mathrm{T}$ & $\mathrm{T}$ & $\mathrm{H}$ & $\mathrm{P}$ & $\mathrm{A}$ & $\mathrm{D}$ & $\mathrm{C}$ & $\mathrm{E}$ & $\mathrm{T}$ & $\mathrm{P}$ & $\mathrm{P}$ & $\mathrm{P}$ & $\mathrm{F}$ \\
\hline PPV6/GX18 & Q & $\mathrm{G}$ & $\mathrm{P}$ & I & $\mathrm{H}$ & $\mathrm{R}$ & $\mathrm{D}$ & $\mathrm{L}$ & $\mathrm{V}$ & $\mathrm{S}$ & $\mathrm{D}$ & $\mathrm{C}$ & $\mathrm{C}$ & $\mathrm{T}$ & $\mathrm{P}$ & $Q$ & $\mathrm{P}$ & A & $\mathrm{E}$ & $\mathrm{C}$ & $\mathrm{Q}$ & $\mathrm{T}$ & $\mathrm{P}$ & $\mathrm{P}$ & $\mathrm{P}$ & $\mathrm{F}$ \\
\hline PPV6/GX22 & $\mathrm{H}$ & $\mathrm{G}$ & $\mathrm{H}$ & $\mathrm{I}$ & $\mathrm{R}$ & $\mathrm{R}$ & $\mathrm{D}$ & $\mathrm{L}$ & $\mathrm{V}$ & $\mathrm{S}$ & D & $\mathrm{C}$ & $\mathrm{C}$ & $\mathrm{T}$ & $\mathrm{T}$ & $\mathrm{Q}$ & $\mathrm{P}$ & $\mathrm{A}$ & D & $\mathrm{C}$ & $\mathrm{Q}$ & $\mathrm{T}$ & $\mathrm{P}$ & $\mathrm{P}$ & $\mathrm{P}$ & $\mathrm{F}$ \\
\hline PPV6/GX33 & $\mathrm{H}$ & $\mathrm{G}$ & $\mathrm{H}$ & I & $\mathrm{R}$ & $\mathrm{R}$ & $\mathrm{D}$ & $\mathrm{L}$ & $\mathrm{A}$ & $\mathrm{S}$ & $\mathrm{D}$ & $\mathrm{C}$ & $\mathrm{C}$ & $\mathrm{T}$ & $\mathrm{T}$ & Q & $\mathrm{P}$ & $\mathrm{P}$ & $\mathrm{D}$ & $\mathrm{C}$ & $\mathrm{Q}$ & $\mathrm{T}$ & $\mathrm{P}$ & $\mathrm{P}$ & $\mathrm{P}$ & $\mathrm{F}$ \\
\hline PPV6/GX41 & $\mathrm{H}$ & $\mathrm{G}$ & $\mathrm{H}$ & I & $\mathrm{R}$ & $\mathrm{H}$ & $\mathrm{D}$ & $\mathrm{L}$ & $\mathrm{V}$ & $\mathrm{T}$ & $\mathrm{D}$ & $\mathrm{C}$ & $\mathrm{F}$ & $\mathrm{A}$ & $\mathrm{T}$ & $Q$ & $\mathrm{P}$ & $\mathrm{A}$ & $\mathrm{D}$ & $\mathrm{C}$ & $\mathrm{E}$ & $\mathrm{T}$ & $\mathrm{P}$ & $\mathrm{P}$ & $\mathrm{P}$ & $\mathrm{F}$ \\
\hline PPV6/GX44 & $Q$ & $\mathrm{G}$ & $\mathrm{P}$ & $\mathrm{I}$ & $\mathrm{R}$ & $\mathrm{R}$ & $\mathrm{V}$ & $\mathrm{F}$ & $\mathrm{V}$ & $\mathrm{S}$ & $\mathrm{G}$ & $\mathrm{R}$ & $\mathrm{C}$ & $\mathrm{A}$ & $\mathrm{T}$ & $Q$ & $\mathrm{H}$ & $\mathrm{A}$ & $\mathrm{D}$ & $\mathrm{C}$ & $\mathrm{E}$ & $\mathrm{T}$ & $\mathrm{P}$ & $\mathrm{P}$ & $\mathrm{P}$ & $\mathrm{F}$ \\
\hline PPV6/GX51 & $\mathrm{H}$ & $\mathrm{G}$ & $\mathrm{H}$ & I & $\mathrm{R}$ & $\mathrm{R}$ & $\mathrm{D}$ & $\mathrm{L}$ & $\mathrm{V}$ & $\mathrm{S}$ & D & $\mathrm{C}$ & $\mathrm{C}$ & $\mathrm{T}$ & $\mathrm{T}$ & $\mathrm{Q}$ & $\mathrm{P}$ & A & D & $\mathrm{C}$ & $\mathrm{E}$ & $\mathrm{T}$ & $\mathrm{P}$ & $P$ & $\mathrm{P}$ & $F$ \\
\hline
\end{tabular}

Figure 5

Amino acid mutation analysis of the isolates. a. Map detail the conserved and variable region of PPV6. Conserved regions (164-256, 414-787, and 1090-1190) are highlighted in green, the variable region (1163 ) is highlighted in blue and the highly variable region (103-133) is highlighted with orange outlined in black. b. Amino acid substitution in putative VP1 conserved regions of the PPV6 isolates.

\section{Supplementary Files}

This is a list of supplementary files associated with this preprint. Click to download.

- SupplementaryFigure1.pdf

- SupplementaryFigure2.pdf 
- Tables1.docx

- TableS2.docx

- Tables3.docx 